\title{
Aplicación de la escala de gravedad de lesiones genitales en víctimas de violencia sexual
}

\section{Application of the genital injury severity scale in sexual violence victims}

1 María de los Ángeles Galarza Pazmiño iD https://orcid.org/0000-0003-4001-3458 Médico Especialista en Medicina Forense, Magister en Criminalística, Médico Perito angelesgalarzapazmino@gmail.com

2 Jorge Marcelo Quintana Yánez id https://orcid.org/0000-0001-7918-556X Médico Especialista en Medicina Forense, Magister en Criminalística, Médico Perito marcelo.quintana.y@gmail.com

3 Verónica Paulina Cáceres Manzano https://orcid.org/0000-0001-9560-9625 MSc. en Criminalística, Perito en Biología, Docente Universitario, Grupo de Investigación “Análisis de Muestras Biológicas y Forenses", Universidad Nacional de Chimborazo, Docente Facultad de Ciencias de la Salud, km 3/1/2 Vía Guano Campus Edison Rivera, Riobamba. vcaceres@unach.edu.ec

4 Fausto Patricio Olivo Cerda https://orcid.org/0000-0001-8706-8021 Especialista en Ciencias Forenses, Experto en Criminalística, Identidad Humana, Balística y Documentología. Director de la Unidad de Delitos contra la Vida, Muertes Violentas, Desapariciones, Extorsión y Secuestro de la Policía Nacional, Ecuador.

fausolivo@gmail.com

5 María Eugenia Lucena de Ustáriz (iD https://orcid.org/0000-0001-9120-345X Ph.D. en Ingeniería de Procesos, Universidad Nacional de Chimborazo, Grupo de Investigación "Análisis de Muestras Biológicas y Forenses", Universidad Nacional de Chimborazo, Docente Facultad de Ciencias de la Salud, km 3/1/2 Vía Guano Campus Edison Rivera, Riobamba. mlucena@unach.edu.ec

Artículo de Investigación Científica y Tecnológica Enviado: $27 / 03 / 2022$

Revisado: $09 / 04 / 2022$

Aceptado: 19/05/2022

Publicado:22/06/2022

DOI: https://doi.org/10.33262/anatomiadigital.v5i2.1679

Cítese:

Galarza Pazmiño, M. de los Ángeles, Quintana Yánez, J. M., Cáceres Manzano, V. P., Olivo Cerda, F. P., \& Lucena de Ustáriz, M. E. (2022). Aplicación de la escala de gravedad de lesiones genitales en víctimas de violencia sexual. Anatomía Digital, 5(2), 125-138. https://doi.org/10.33262/anatomiadigital.v5i2.1679

ANATOMÍA DIGITAL, es una Revista Electónica, Trimestral, que se publicará en soporte electrónico tiene como misión contribuir a la formación de profesionales competentes con visión humanística y crítica que sean capaces de exponer sus resultados investigativos y científicos en la misma medida que se promueva mediante su intervención cambios positivos en la sociedad. https://anatomiadigital.org 


\section{ADÉnigital}

\section{Palabras} claves: violencia, sexual, observación, examen forense, medicina legal

\section{Keywords:}

violence, sexual, naked eye, forensic examination, medicine legal.

\section{Resumen}

Introducción: La violencia de tipo sexual puede afectar a todo ser humano sin importar edad, sexo, etnias, condición social o laboral; un punto importante a recalcar es que afecta a la libertad de decisión y de consentimiento expreso del goce de los derechos e integridad sexual de quien se está formando y no tiene la capacidad de decidir sobre actividades sexuales de manera libre. Según el Instituto Nacional de Estadísticas y Censos en el año 2011, el 25,7\% de mujeres ecuatorianas han sido víctimas de violencia sexual. Dentro de la exploración física de la víctima es importante detectar lesiones a nivel extragenital, paragenital y/o genital; para ayudar en el proceso se han creado escalas de aplicación durante el examen sexológico forense, una de estas es la Escala de Gravedad de Lesiones Genitales (GISS: Genital Injury Severity Scale) desarrollada por Kelly et al. en el año 2013, para valorar y dar una gradación a las lesiones en el área genital de las víctimas femeninas. Objetivo: Aplicar la Escala de Gravedad de Lesiones Genitales (GISS) y relacionarla con la observación directa durante el examen sexológico forense. Metodología: El diseño del estudio fue observacional, transversal y analítico, en mujeres víctimas de presunta agresión sexual que se les realizó el examen sexológico forense. Resultados: Se demostró que existe relación entre la escala de gravedad de lesiones genitales (GISS) y la observación directa (OD) (Índice de Kappa: 0,66; IC95\%: 0,52-0,79) para establecer lesiones genitales en mujeres víctimas de presunta agresión sexual. Conclusiones: La Escala de gravedad de lesiones genitales (GISS) no presenta ventajas de identificación de lesiones con respecto a la observación directa.

\section{Abstract}

Introduction: Sexual violence can affect every human being regardless of age, sex, ethnicity, social or employment status; It affects the freedom of decision and express consent to the benefit of sexual rights and the sexual integrity of those who are being prepared and who cannot the ability to decide on sexual activities deliberately. According to the National Institute of Statistics and Censuses in 2011, 25.7\% of Ecuadorian women have been victims of sexual violence. Within the physical examination of the victim, it is important to detect extra-genital, para-genital and/or genital 
injuries; To assist in the process, application scales have been created during the forensic sexological examination, one is the Genital Injury Severity Scale (GISS) developed by Kelly et al. in 2013, to assess and grade the injuries in the genital area of female victims. Objective: To apply the Genital Injury Severity Scale (GISS) and relate it to the naked eye during the forensic sexological examination. Methodology: The study design was observational, cross-sectional, and analytical, in women victims of alleged sexual assault who underwent a forensic sexological examination. Results: It was shown that there is a good diagnostic concordance to establish genital injuries in women victims of alleged sexual assault, between the genital injury severity scale (GISS) and the naked eye (NE) (Kappa index: 0.66; 95\% CI: 0.520.79). Conclusions: The genital injury severity scale has a diagnostic agreement for the detection of injuries in the genital area concerning direct observation.

\section{Introducción}

La violencia de tipo sexual puede afectar a todo ser humano sin importar edad, sexo, etnias, condición social o laboral; afecta a la libertad de decisión y de consentimiento expreso del goce de los derechos y la integridad sexuales de quien se está formando y que no tiene la capacidad de decidir sobre actividades sexuales de manera libre.

La Organización Mundial de la Salud (OMS) (1), define a la violencia sexual como "la tentativa de consumar un acto sexual, los comentarios y las insinuaciones sexuales no deseados, o las acciones para comercializar o utilizar de cualquier otro modo la sexualidad de una persona mediante coacción por otra persona, independientemente de la relación de esta con la víctima, en cualquier ámbito, incluido el hogar y el lugar de trabajo".

La víctima que busca asistencia concurre a entidades de salud, policiales y/o judiciales y el personal que va a tener el primer contacto debe poseer capacitación técnica especializada para una valoración integral y oportuna que a posterior de respuesta a los requerimientos judiciales (2).

La evaluación médica forense en caso de agresión de tipo sexual provee evidencia del hecho delictivo que a posterior facilitará la identificación, enjuiciamiento, condena y castigo de los agresores (2); y la reparación integral de la víctima. 


\section{ADEtigital}

Una parte fundamental es el examen sexológico forense, con el que se da la individualización de lesiones con su respectivo tratamiento y la recolección de indicios (muestras biológicas) (3). Hay que tener en cuenta que, a pesar de la atención adecuada, existen casos donde las lesiones en el área genital no se observan a simple vista, por factores propios de la víctima como el encontrarse bajo efecto de sustancias que imposibiliten oponerse al ataque o medios de coacción (4).

Es importante valerse de técnicas o herramientas auxiliares como la colposcopía y azul de toluidina que de manera aislada o combinada elevan la detección de las lesiones, estos medios adyuvantes no se han extendido en nuestro medio y de manera general se cumple con la observación directa, a pesar de que existe evidencia que la frecuencia de detección de lesiones se incrementa; es así que se han desarrollado escalas de valoración conjunta como la aplicada en el presente estudio que corresponde a la Escala de Gravedad de Lesiones Genitales (Genital Injury Severity Scale: GISS), en donde se analiza de manera focalizada estructuras del área genital femenina, las lesiones presentes derivadas de una relación sexual según su gravedad.

\section{Fundamento Teórico}

La violencia sexual es toda acción de naturaleza sexual o su tentativa, que repercutirán en la salud física y mental de las víctimas (5).

La sexología es la disciplina que estudia las cuestiones vinculadas al sexo en sus vertientes médico, sociológico, jurídico y antropológico y la Sexología Forense es la rama de la Medicina Legal con la que se aplica los conocimientos médicos para dar soluciones de los problemas jurídicos que se plantean en torno al sexo y uno de ellos corresponde a los delitos sexuales $(6,7)$.

El cuerpo humano para el estudio médico legal pericial, en casos de delitos sexuales se divide en tres áreas topográficas:

A. Área genital: que incluye genitales externos, la región anorrectal y periné.

B. Área paragenital, que comprende la zona abdominal infraumbilical, la cara interna de la raíz de ambos muslos y los glúteos.

C. Área Extragenital: abarca el resto de la topografía, de mayor importancia cuello, mamas, muñecas y tobillos $(8,9)$.

La exploración de la víctima incluye las lesiones corporales, por mínimas que sean pueden corroborar los acontecimientos relatados por la víctima e indican el grado de violencia física utilizada (10).

En términos medicolegales la región anal es considerada como parte del área genital, la examinación tanto de la parte ginecológica, como proctológica incluye que la víctima se 
coloque en dos posiciones; primero que se encuentre acostada en la mesa ginecológica, en posición de litotomía y segundo en posición genupectoral, así se logrará visualizar todas las estructuras anatómicas mencionadas y verificar la presencia o ausencia de lesiones (9). Las lesiones encontradas se consideran de tipo agudo cuando han sido ocasionadas en un tiempo menor de ocho días (10).

Para la elaboración de la Escala de Gravedad de Lesiones Genitales (GISS), el primer estudio fue efectuado por Larkin et al. en el 2012 (11), que comparó las lesiones producto de una agresión sexual con aquellas de relaciones sexuales consensuales, se analizó tanto la prevalencia como la gravedad de la lesión genital, demostrándose que las mujeres con reporte de agresión sexual reciente tenían lesiones más severas en relación con las relaciones consensuales (11). El siguiente estudio desarrollado por Kelly et al. en el 2013 (12), que realizó una medición basado en la gravedad de las lesiones genitales externas, sean de una agresión sexual o una relación consentida, usando diferentes variables con empleo de un lente de amplificación y el azul de toluidina, se formó un instrumento de doce factores que miden cinco tipos de gravedad y dos clases de gravedad con el que se logró diferenciar a las pacientes de la cohorte de agresión sexual con las de relaciones consensuales $(\mathrm{p}<0,0001$ es decir que esta herramienta logra discriminar las dos cohortes y esta relación de variables es estadísticamente significante); esta escala no responde a la cuestión de la culpabilidad o la inocencia de una persona que solo le corresponde a un juez (12).

Tabla 1. Escala de Gravedad de Lesiones Genitales (GISS)

\begin{tabular}{|c|c|c|c|c|c|}
\hline \multicolumn{6}{|c|}{$\begin{array}{l}\text { ESCALA DE GRAVEDAD DE LESIONES GENITALES } \\
\text { GENITAL INJURY SEVERITY SCALE }\end{array}$} \\
\hline & Tipo 1 & Tipo 2 & Tipo 3 & Tipo 4 & Tipo 5 \\
\hline Inflamación & $\begin{array}{l}(-) \\
(0)\end{array}$ & $\begin{array}{c}+ \\
\text { Medio } \\
(1)\end{array}$ & & $\begin{array}{c}++ \\
\text { Significante } \\
\text { (2) }\end{array}$ & \\
\hline $\begin{array}{l}\text { Cambios de } \\
\text { color (eritema o } \\
\text { contusión) }\end{array}$ & $\begin{array}{l}(-) \\
(0)\end{array}$ & $\begin{array}{c}+ \\
\text { Rosado /Rojo } \\
(1)\end{array}$ & Eritema & ficante & $\begin{array}{l}\text { Violáceo } \\
\text { (3) }\end{array}$ \\
\hline $\begin{array}{l}\text { Lesión Tisular } \\
\text { (abrasión hasta } \\
\text { desgarro) }\end{array}$ & $\begin{array}{l}(-) \\
(0)\end{array}$ & $\begin{array}{l}\text { Superficial: } \\
\text { visualizado con } \\
\text { colposcopio o } \\
\text { azul de } \\
\text { toluidina } \\
\text { (1) }\end{array}$ & $\begin{array}{c}\text { Medio: } \\
\text { Abrasiones } \\
\text { lineales en la } \\
\text { epidermis bajo } \\
\text { inspección } \\
\text { (2) }\end{array}$ & $\begin{array}{c}\text { Moderado: } \\
\text { Abrasiones } \\
\text { profundas y } \\
\text { anchas en la } \\
\text { epidermis } \\
\text { (3) }\end{array}$ & $\begin{array}{c}\text { Severo: } \\
\text { Desgarro hasta } \\
\text { tejido } \\
\text { subcutáneo o } \\
\text { más profundo } \\
\text { (4) }\end{array}$ \\
\hline $\begin{array}{l}\text { Himen e introito } \\
\text { vaginal }\end{array}$ & & $\begin{array}{l}(-) \\
(0)\end{array}$ & $\begin{array}{l}\text { Desgarro } \\
\text { incompleto } \\
\text { (2) }\end{array}$ & $\begin{array}{c}\text { Desgarro } \\
\text { completo } \\
\text { (3) }\end{array}$ & $\begin{array}{c}\text { Desgarro } \\
\text { completo } \\
\text { extiende hasta el } \\
\text { introito } \\
\text { (4) }\end{array}$ \\
\hline
\end{tabular}


Tabla 1. Escala de Gravedad de Lesiones Genitales (GISS) (continuación)

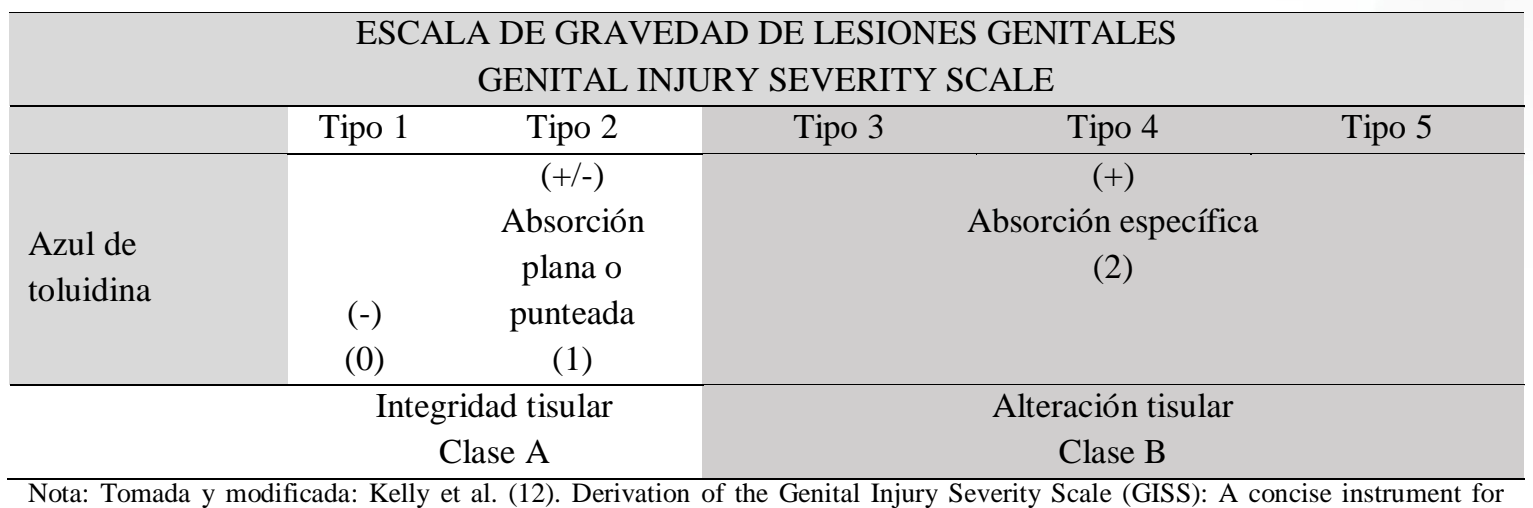

description and measurement of external female genital injury after sexual intercourse.

En la examinación se utilizó el azul de toluidina que se aplicó en los labios menores y en la horquilla posterior, el exceso fue removido con un lubricante a base de agua, se toma una imagen amplificada con el uso del colposcopio y se aplicó la escala de gravedad de lesiones genitales tomando en cuenta estructuras anatómicas cómo: labios menores, clítoris o área adyacente, tejido peri uretral o meato uretral; vestíbulo, himen, fosa navicular y horquilla posterior; e identifica cinco criterios 1) Inflamación (hinchazón) 2) Cambios de color 3) lesión tisular en labios menores u horquilla posterior 4) Lesión del himen 5) Absorción de azul de toluidina por el tejido. El resultado son cinco tipos de hallazgos (1-5), que cuando las características de estos se comparten entre dos tipos, esto se agrupan en clases (A y B) $(11,12)$.

El azul de toluidina es un colorante, acidófilo, metacromático y tiñe los núcleos celulares de azul (13). El estrato superficial de la piel de la vulva normal no contiene núcleos y las laceraciones, exponen las capas profundas de la piel donde las células poseen núcleo y se encuentran en proceso de maduración (14).

Esta sustancia cuando la capa de la piel de células no nucleadas que han sido retiradas tiñe a las células nucleadas subyacentes. Aunque no es un estándar uniforme de la atención, el tinte puede mejorar la capacidad del examinador para visualizar las lesiones genitales. La identificación de estas lesiones puede corroborar evidencia de relaciones sexuales no consentidas. La técnica es aplicada cuando se ha completado otros procedimientos, se aplica al $1 \%$ de solución acuosa de azul de toluidina y se limpia el exceso con un algodón y gel lubricante. La aplicación de esta técnica incrementa la detección de lesiones del 16\% al 40\% (15).

\section{Metodología}

Se planteó estudio con diseño observacional de tipo transversal donde se estableció la relación de concordancia de dos pruebas diagnósticas como son la observación directa y 


\section{ADÉnigital}

la escala de gravedad de lesiones genitales (GISS), aplicado en mujeres mayores de doce años que acudieron a la unidad de flagrancia en Quito, a quienes mediante aceptación y firma de consentimiento informado se les explico la aplicación de la escala logrando una muestra de cincuenta mujeres. Se recolectó los datos mediante entrevista directa y la inspección durante el examen sexológico forense y posterior aplicación de la escala de gravedad de lesiones genitales; datos que fueron registrados y posterior organizados en una tabla por categorías en el Programa Microsoft Excel 1803 correspondiente al paquete de Microsoft Office 365, ulterior a su revisión se creó una base de datos que fue codificada para posteriormente ser exportada y analizada en el paquete estadístico SPSS versión de prueba obteniéndose estadística para análisis y se aplicó el Coeficiente kappa de Cohen, para determinar la concordancia entre métodos diagnósticos.

\section{Resultados}

Se obtuvieron datos de cincuenta mujeres mayores de doce años que acudieron a la Unidad de Flagrancia Quito, con una edad promedio 19 años, la edad mínima 12 años y máxima 38 años, siendo las más afectadas las mujeres de 15 años, solteras en el 82\%. El nivel de instrucción fue básico en $42 \%$, bachilleres en $40 \%$ y educación superior en el $8 \%$; la ocupación más frecuente fue estudiantes en el $70 \%$.

Los hechos y la relación con el examen ginecológico forense, el 52\% de las víctimas acudieron en las primeras 24 horas, refiriendo algún tipo de intimidación o amenaza en el $26 \%$, con relato de relación forzada en el $64 \%$, vía de penetración a través de vagina en el $48 \%$, con el miembro viril en el $52 \%$ de los casos. El uso de alcohol y/o drogas previo al evento en un $72 \%$ de los casos, lo que impidió un relato adecuado de la víctima.

En la valoración del himen, la morfología anular predominó siendo el 50\%, reducido a carúnculas mirtiformes con el $24 \%$ y $16 \%$ festoneado; con menor frecuencia el labiado $(4 \%)$, semilunar $(4 \%)$ y lobulado $(2 \%)$. La presencia del himen dilatable se presentó en el 30\%. La presencia de desgarros se encontró en el 42\%; antiguos en el 85,7 \% y recientes en el $14,3 \%$.

La valoración de lesiones en el área genital (excluyendo himen y ano), presentó el 58\% indemnidad del área y en el $42 \%$ que se encontró lesiones (desgarro reciente $48 \%$, signos de inflamación $28 \%$ y laceración $14 \%$ ).

En el himen, el 50\% de los casos presentó indemnidad de la zona y de los casos restantes, predominó el $64 \%$ de desgarro antiguo, recalcando que un $8 \%$ compartía está característica más algún signo de inflamación sea rubor o tumefacción y en el $12 \%$ se presenta un desgarro reciente. 


\section{ADEtigital}

Para la región anal el $76 \%$ de los casos reportó indemnidad de la zona, del porcentaje que presentó lesiones: el 66,8\% corresponde a desgarro reciente, esfínter anal dilatado en el 16,6\% y margen anal equimótico en el 16,6\%.

La observación directa, es una técnica, en la que mediante la visualización se inspecciona la zona genital, sin ningún método auxiliar, en el $54 \%$ de las investigadas mantenía indemnidad corporal, el $12 \%$ con eritema o rubor y tumefacción que se agruparon en signos de inflamación y además de un porcentaje similar para compromiso de las capas superficiales con equimosis y laceración; y del 22\% con desgarro; sea de piel o de la mucosa.

En la descripción del área paragenital existió un 92\% de indemnidad y de las lesiones descritas el $75 \%$ correspondía a equimosis. En el área extragenital, las lesiones correspondían al 60\%, distribuidas en diferentes partes del cuerpo.

Para el análisis de la escala de gravedad de lesiones genitales (GISS) en el 56\% se encontró negatividad para lesiones (Tipo 1: no hallazgos), $8 \%$ de lesión leve, con signos de inflamación como eritema y/o tumefacción (Tipo 2: hallazgos no específicos), 26\% como lesión moderada, que está ocupando las capas superficiales de la epidermis sea equimosis o laceración (Tipo 3: hallazgos menos severos); y 10\% para lesión grave a que corresponde a la laceración (Tipo 4: hallazgos graves); no se encontró el Tipo 5 (lesión muy grave o severa).

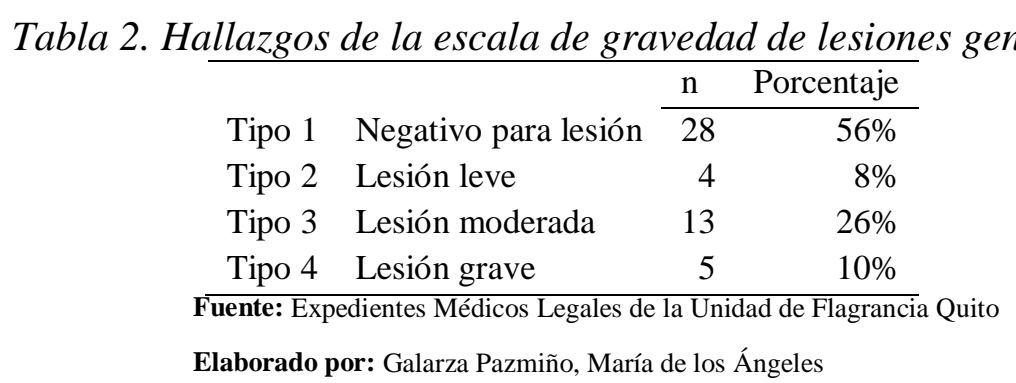

La coloración con azul de toluidina del área genital, tinción negativa corresponde al $58 \%$ de la muestra, una tinción plana o punteada del $14 \%$ y la absorción específica del $28 \%$.

En relación con la concordancia diagnóstica para determinar lesiones genitales en mujeres víctimas de presunta agresión sexual, se encontró una muy buena concordancia entre la observación directa frente a la tinción de toluidina (Índice de Kappa: 0,83; IC95\%: 0,68-0,99), tabla 3. 
Tabla 3. Concordancia diagnóstica entre $O D^{\mathscr{I}}$ y tinción con azul de toluidina

\begin{tabular}{|c|c|c|c|}
\hline \multirow{4}{*}{ 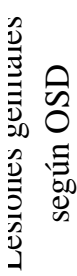 } & \multicolumn{3}{|c|}{ Lesiones genitales según la tinción con azul de toluidina } \\
\hline & & PRESENTE & AUSENTE \\
\hline & PRESENTE & 20 & 3 \\
\hline & AUSENTE & 1 & 26 \\
\hline
\end{tabular}

Índice de Kappa: 0,83 (IC95\%: 0,68-0,99)

Fuente: Expedientes Médico Legales de la Unidad de Flagrancia Quito

Elaborado por: Galarza Pazmiño, María de los Ángeles OD Observación directa

En relación con la concordancia diagnóstica para determinar lesiones genitales en mujeres víctimas de presunta agresión sexual, se encontró una buena concordancia entre ambos métodos evaluados (Índice de Kappa: 0,66; IC95\%: 0,52-0,79). Remítase a la tabla 4 y gráfica 2.

Tabla 4. Concordancia diagnóstica entre la OD" y la GISS*

\begin{tabular}{|c|c|c|c|}
\hline \multirow{4}{*}{ 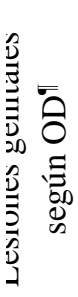 } & \multicolumn{3}{|c|}{ Escala de gravedad de lesiones genitales (GISS*) } \\
\hline & & & AUSENTE \\
\hline & PRESENTE & 21 & 2 \\
\hline & AUSENTE & 1 & 26 \\
\hline
\end{tabular}

Índice de Kappa: 0,66 (IC95\%: 0,52-0,79)

Fuente: Expedientes Médico Legales de la Unidad de Flagrancia Quito

Elaborado por: Galarza Pazmiño, María de los Ángeles OD Observación simple directa

*Escala de gravedad de lesiones genitales (Genital injury severity scale)

\section{Discusión}

La dinámica de una relación sexual es inconstante y depende de factores del sujeto activo, pasivo y el uso de medios violentos (9), las más afectadas son mujeres de 15 años, siendo un factor de riesgo para sufrir lesiones durante la agresión; predominan las estudiantes $(70 \%)$, se correlaciona con el estudio brasileño, donde la mayoría tienen bajos niveles educacionales en el $86,9 \%$.

En el 94\% de los casos se les practicó el examen sexológico forense en las primeras 72 horas, al $52 \%$ en las primeras 24 horas, con lo que se consigue una tomas de muestras adecuadas, un $64 \%$ refirió que fue una relación forzada, la vía de penetración fue 


\section{ADEtigital}

vaginal $48 \%$, anal $2 \%$, vaginal - anal $10 \%$ y desconocían que les paso en el $40 \%$, según Scherer et al. (16), que excluyó a las víctimas que han consumido alcohol, reportó que la vía de penetración fue vaginal $66 \%$, anal $2 \%$, vaginal - anal 11\%. El uso de algún tóxico fue de un 72\% (alcohol: 54\%, otras drogas: 18\%), los efectos de las sustancias hacen que se pierdan un sinnúmero de datos; en otro estudio el 61,8\% presentó consumo de alcohol, que implico una menor frecuencia de lesiones, además que significa un riesgo mayor de sufrir una agresión sexual $(17,18)$.

El himen es considerado como "barrera anatómica y frontera jurídica en la comisión del delito contra la libertad sexual" (19); y en otros estudios la vagina anatómica (tubo muscular) versus la legal (limita entre la vulva y el cérvix) la penetración no involucra al himen (13).

La observación directa se realiza mediante la inspección, para detectar lesiones o indemnidad (4), en área genital el 54\% con indemnidad y $46 \%$ con lesiones: signos de inflamación (12\%), equimosis - laceración en $12 \%$ y desgarro en $22 \%$ compromiso de todo el espesor con desgarro; para Zilkens et al. (4), se excluyeron hallazgos del tipo eritema o tumefacción, por ser considerados como inespecíficos en la definición de lesión genital encontrando lesiones en el 52,7\% de los casos, porcentaje mayor al del presente estudio, pero esta concepción dista mucho del estudio hecho por Hinojosa (20), donde la mayor parte de reportes en los expedientes médicos fue el de eritema o enrojecimiento.

Explicar desde el plano corporal un ataque sexual, es complejo, es más viable demostrar lesiones para continuar con un proceso penal con pruebas objetivas, pero muchas ocasiones la coerción provoca terror psicológico (13), con incapacidad de defenderse (no lesiones) o a posterior omitir datos por vergüenza o humillación post agresión, miedo a represalias y preocupación de la reacción de las personas cercanas (21), o de la dicotomía descrita por Scherer et al. (16), en la lesión genito-anal de una agresión sexual, que la mayoría van a presentar pocas lesiones y pocas van a presentar muchas lesiones; una máxima en medicina legal es que la ausencia de lesiones no excluye el ataque sexual (18).

La escala de gravedad de lesiones genitales (GISS) estandariza una serie de parámetros; para clasificar las lesiones que se encuentran en una relación sexual consentida y las de una agresión; al aplicar el azul de toluidina (AT), en mujeres con relaciones consentidas $(\mathrm{n}=98)$ las lesiones descritas como laceraciones se presentaron en el 50\%, en comparación con la observación del $31 \%$ y en el grupo casos (agresión sexual, n=39) con coloración fue del 38\% y la observación del 28\% (22), a pesar de las diferencias en la detección, los resultados no son concluyentes y dependen de factores de la víctima y del examinador (23). 


\section{ADEtigital}

Se realizó la concordancia diagnóstica entre la observación directa con la escala (GISS) y con la coloración (AT), encontrando una concordancia buena (Índice de Kappa: 0,66; IC95\%: 0,52-0,79) y muy buena (Índice de Kappa: 0,83; IC95\%: 0,68-0,99), respectivamente; es decir que si la observación detecta lesiones la GISS lo hace de manera similar y el azul tiñe las mismas; sí la observación corrobora la indemnidad genital, la GISS señala hallazgos negativos y no se da la absorción del azul por el tejido, porque este se encuentra íntegro.

En torno a la severidad de las lesiones la misma no indica si fue o no un acto sexual voluntario (16), existe variedad de factores que actúan como el uso de alcohol o la coerción.

Benson A. refiere que sí como profesionales tenemos el objetivo de garantizar la calidad de la evidencia, es nuestro deber desarrollar un lenguaje consistente, un método de detección y una estrategia de medición para ayudar al sistema de justicia (24).

\section{Conclusiones}

- Existe buena correlación diagnóstica usando la Observación directa y las Escala de Gravedad de Lesiones Genitales (GISS).

- Las lesiones genitales son muy diversas y pueden ir desde la tumefacción o enrojecimiento (eritema) hasta el desgarro cutáneo - mucoso, sin lograr establecer patrones en caso de agresión.

- Las lesiones corporales pueden ayudar a corroborar el relato de la víctima, tomar en consideración que la indemnidad corporal no desmiente los hechos y que puede coexistir una ambigüedad, como es presencia de lesiones genitales e indemnidad corporal y viceversa.

- De existir indemnidad corporal prestar especial atención con el uso de tóxicos o medios de coerción como la intimidación o amenaza.

\section{Referencias Bibliográficas}

Organización Mundial de la Salud [OMS] y Escuela de Higiene y Medicina Tropical de Londres. Prevención de la violencia sexual y violencia infligida por la pareja contra las mujeres: qué hacer y cómo obtener evidencias Washington DC: Organización Panamericana de la Salud; 2011.

Arroyo Sánchez G. Valoración médico legal de la víctima de delito sexual.

Sommer M, Brunner L, Brown K, Buschur, Everett J, Fargo J, et al. Injuries from Intimate Partner and Sexual Violence: Significance and Classification 


\section{ADÉnigital}

Systems. J Forensic Leg Med. 2012; 19(5): p. 250-263.

Zilkens R, Smith D, Phillips M, Mukhtar A, Semmens J, Kelly M. Genital, and anal injuries: A cross-sectional Australian study of 1266 women alleging recent sexual assault. Forensic Science International. 2017;(275): p. 195-202.

Organización Panamericana de la Salud. Informe mundial sobre la violencia y la salud: Resumen. Washington: Organización Mundial de la Salud, Oficina Regional para las Américas. ISBN 9241545623.

Berro G. Sexología Médico Legal. En Berro G, Adriasola G, Albínez J, Alterwain P, Ayala W, Burgues S, et al. Medicina Legal Derecho Médico y Aspectos Bioéticos. Montevideo: Fundación Cultura Universitaria; 2013. p. 205-219.

Patitó J. Sexología MédicoLegal. En Vicino C, editor. Medicina Legal. Buenos Aires: Ediciones Centro Norte; 2000. p. 315-326.

Kvitko L. El Himen Beenos Aires: Ediciones la Rocca; 2005.

Vargas Alvarado E. Delitos contra la libertad sexual. En Vargas Alvarado E. Medicina Legal. México: Editorial Trillas; 2002. p. 251-264.

Castel González B, Bernad Pérez ML. Consideraciones médico-legales en torno a las agresiones sexuales: puesta al día e importancia de las características lesionales de las víctimas. Trabajo Fin de Grado. Zaragoza: Universidad de Zaragoza, Facultad de Medicina.

Larkin H, Cosby C, Kelly, Paolinetti L. A pilot study to test the differential validity of a genital injury severity scale, in development for use in forensic sexual assault examinations. Journal of Forensic Nursing. 2012; 8: p. 20-38.

Kelly D, Larkin H, Cosby C, Paolinetti L. Derivation of the Genital Injury Severity Scale (GISS): A concise instrument for description and measurement of external female genital injury after sexual intercourse. Journal of Forensic and Legal Medicine. 2013; 20(1): p. 724-731.

White C. Genital injuries in adults. Best Practice \& Research Clinical Obstetrics and Gynaecology. 2013; 27.

Lauber A, Souma M. Use de toluidine blue for documentation of a traumatic intercourse. Obstetric Gynecol. 1982; 60(644): p. 644 - 648. 
Sachs C, Wheeler M. Examination of the Sexual Assault Victim. En Roberts J, Hedges J. Clinical Procedures in Emergency Medicine. Philadelphia: Saunders; 2010. p. 1074-1075.

Scherer S, Hansen S, Lynnerup N. Discrepancy between information reported by the victims of sexual assaults and clinical forensic findings. Dan Med J. 2014; 61(9).

Hilden, Schei, Sidenius. Genito anal injury in adult female victims of sexual assault. Forensic Science International. 2005; 154.

Maguire, Goodall, Moore. Injury in adult female sexual assault complainants and related factors. European Journal of Obstetrics \& Gynecology and Reproductive Biology. 2009; 142.

Puma L. Valor probatorio de la colposcopía en el delito de violación sexual en mujeres con himen complaciente, Sicuani, 2016. Tesis especialización. Cusco: Universidad Nacional de San Antonio de Abad del Cusco, Escuela de Postgrado.

Hinojosa K. Tipos de lesiones más frecuente descritos en los certificados ginecológicos realizados en el centro de atención integral Toluca del periodo de septiembre 2011 a septiembre de 2012. Tesis de especialidad. Toluca: Universidad Autónoma de México, Departamento de Evaluación Profesional.

Novo M, Díaz P, Amado B. Efectos de contexto en la formación de juicios en un caso de violación. Anuario de Psicología Jurídica. 2015; 25.

Astrup, Ravn, Thomsen J, Lauritsen. Patterned genital injury in cases of rape. A case - control - study. Journal of Forensic and Legal Medicine. 2013; 20(5).

Laitinen F, Grudmann O, Ernst E. Factor that influence the variability in findings of anogenital injury in adolescent/ adult sexual assault victims. Am J Forensic Med Pathol. 2013; 34(3).

Benson A. Development, Validity and Reliability of the Vulvar Acute Genital Injury (VAGIS) Instrument. Dissertation Doctoral. California: University of California. ProQuest Number: 10600768.

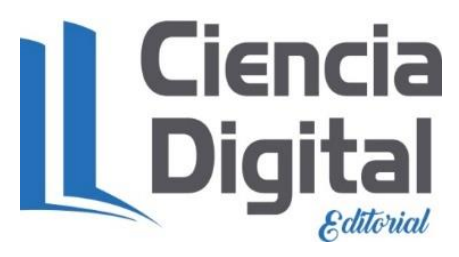


El artículo que se publica es de exclusiva responsabilidad de los autores y no necesariamente reflejan el pensamiento de la Revista Anatomía Digital.

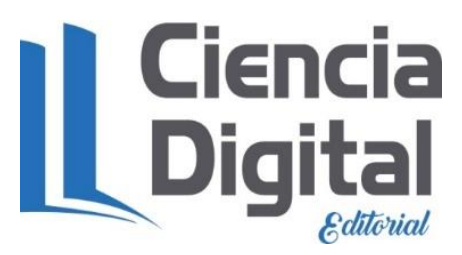

El artículo queda en propiedad de la revista y, por tanto, su publicación parcial y/o total en otro medio tiene que ser autorizado por el director de la Revista Anatomía Digital.
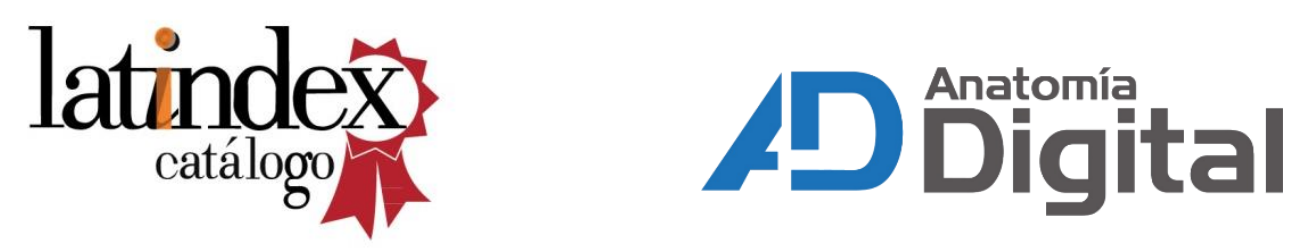

Indexaciones

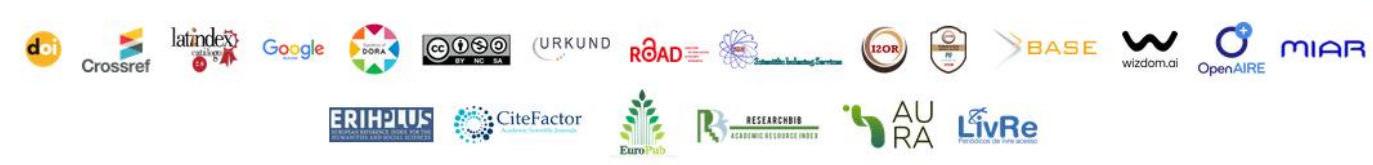

